\title{
A BOUNDARY INTEGRAL EQUATION FOR THE SOLUTION OF A CLASS OF PROBLEMS IN ANISOTROPIC INHOMOGENEOUS THERMOSTATICS AND ELASTOSTATICS*
}

\author{
BY \\ DAVID L. CLEMENTS (University of Adelaide) \\ AND \\ C. ROGERS (University of Waterloo)
}

\begin{abstract}
The solution of an important class of boundary-value problems in anisotropic inhomogeneous thermostatics and elastostatics is obtained in terms of a boundary integral equation. The equation may be used as a basis for the numerical solution of particular boundary-value problems.
\end{abstract}

1. Introduction. The boundary integral equation method is now well established as an extremely useful method for the numerical solution of boundary-value problems governed by linear elliptic partial differential equations with constant coefficients (see for example, Cruse and Lachat [17], Cruse and Rizzo [2]). The method consists of expressing the solution to a particular problem in terms of an integral equation with the integral taken round the boundary of the region under consideration. It is possible to derive this integral equation for a wide class of physical problems. In particular, the boundary integral equation is readily obtained for problems in homogeneous elastostatics and thermostatics where the governing equations are elliptic with constant coefficients. The integral equation is not so easily derived in a usable form for inhomogeneous problems in which the governing equations are still of the elliptic type but with variable coefficients. However, some progress has been made in deriving boundary integral equations for such problems and, in particular, in a recent paper Clements [3] obtained such an equation for a single second-order elliptic equation.

The aim of the present paper is to extend the work of Clements to a more general second-order equation. Specifically, the equation considered by Clements covers a class of inhomogeneous problems in thermostatics and elastostatics for isotropic materials. The present work is concerned with the equation which governs the same class of problems for anisotropic materials.

2. The boundary-value problem. Consider the elliptic partial differential equation

$$
\frac{\partial}{\partial x_{i}}\left[k_{i j} \frac{\partial \phi}{\partial x_{j}}\right]=0,
$$

where the repeated suffix summation convention (summing from 1 to 2 ) is employed. The coefficients $k_{i j}=k_{j i}$ are taken to be functions of $x_{2}$ only. Eq. (2.1) governs the static plane

\footnotetext{
* Received May 10, 1982.
} 
temperature field or antiplane stress field in an anisotropic inhomogeneous material (see Clements [5]). In the first case the $k_{i j}$ denote the heat conduction coefficients and in the second the elastic constants. In either case the coefficients are constrained by physical consideration to be positive and to satisfy the ellipticity condition

$$
k_{12}^{2}-k_{11} k_{22}<0 \text {. }
$$

Here a solution to (2.1) is sought which is valid in a region $R$ in $E^{2}$ with boundary $C$ which consists of a finite number of piecewise smooth closed curves. On $C$ either the dependent variable $\phi$ is specified or $k_{i j}\left(\partial \phi / \partial x_{j}\right) n_{i}$ is specified, where $\mathbf{n}$ denotes the outward pointing normal to $C$. In elasticity $k_{i j}\left(\partial \phi / \partial x_{j}\right) n_{i}$ provides the traction vector and in thermostatics it yields the heat flux.

The method of solution will be to express the solution to the problem in terms of an integral taken round the boundary $C$ of the region $R$ under consideration. In general this integral equation cannot be solved analytically. However, it may be solved numerically by using well-established procedures and hence forms the basis for the numerical solution of particular problems.

3. A general analytical solution to (2.1). The aim in this section is to obtain an analytical solution to (2.1) in terms of an infinite series involving a single arbitrary analytic function. To achieve this aim, consider a representation for the dependent variable $\phi$ in the form

$$
\phi=\sum_{n=0}^{\infty} T_{n}\left(x_{2}\right) E_{n}\left(x_{1}+S\left(x_{2}\right)\right), \quad T_{0} \neq 0,
$$

where the $E_{n}$ satisfy the recurrence relations

$$
E_{n}^{\prime}=E_{n-1} \quad \text { for } n=1,2, \ldots
$$

where the prime denotes the derivative with respect to the argument in question. Substitution shows that if the $\phi$ given by (3.1) is to satisfy (2.1) then the coefficients $T_{n}\left(x_{2}\right)$ and $S\left(x_{2}\right)$ must be such that

$$
\begin{aligned}
\sum_{n=0}^{\infty}\left[\left\{k_{11}+2 k_{12} S^{\prime}+k_{22} S^{\prime 2}\right\}\right. & T_{n} E_{n}^{\prime \prime}+\left\{k_{22}^{\prime} S^{\prime} T_{n}+2 k_{21} T_{n}^{\prime}\right. \\
& \left.\left.+2 k_{22} S^{\prime} T_{n}^{\prime}+k_{22} S^{\prime \prime} T_{n}+k_{21}^{\prime} T_{n}\right\} E_{n}^{\prime}+\left\{k_{22}^{\prime} T_{n}^{\prime}+k_{22} T_{n}^{\prime \prime}\right\} E_{n}\right]=0 .
\end{aligned}
$$

This equation may be rewritten in the form

$$
\begin{aligned}
\sum_{n=0}^{\infty}\left[\left\{k_{11}+2 k_{12} S^{\prime}+k_{22} S^{\prime 2}\right\} T_{n} E_{n}^{\prime \prime}+2\left(k_{12}+k_{22} S^{\prime}\right)^{1 / 2}\right. \\
\left.\cdot \frac{d}{d x_{2}}\left\{\left(k_{12}+k_{22} S^{\prime}\right)^{1 / 2} T_{n}\right\} E_{n}^{\prime}+\frac{d}{d x_{2}}\left(k_{22} T_{n}^{\prime}\right) E_{n}\right]=0 .
\end{aligned}
$$

The first term in (3.6) will be zero for all $n$ if

$$
S^{\prime}=k_{22}^{-1}\left[-k_{12} \pm\left(k_{12}^{2}-k_{11} k_{22}\right)^{1 / 2}\right] \text {, }
$$

while use of (3.2) in the second term together with use of the third term provides

$$
T_{0}=\alpha\left[k_{12}+k_{22} S^{\prime}\right]^{-1 / 2} \quad(\alpha \text { arbitrary constant }),
$$




$$
T_{n}=-\frac{1}{2}\left(k_{12}+k_{22} S^{\prime}\right)^{-1 / 2} \int\left[\frac{\left(d / d x_{2}\right)\left\{k_{22} T_{n-1}^{\prime}\right\}}{\left(k_{12}+k_{22} S^{\prime}\right)^{1 / 2}}\right] d x_{2} \text { for } n=1,2, \ldots
$$

In view of (2.2) it follows that (3.4) yields a complex conjugate pair $\tau\left(x_{2}\right)$ and $\bar{\tau}\left(x_{2}\right)$ where $\tau\left(x_{2}\right)$ is obtained from (3.4) by taking the positive sign. The corresponding $T_{n}$ obtained from (3.5) and (3.6) will be denoted by $T_{n}$ and $\bar{T}_{n}$ respectively. Hence a real function $\phi$ which satisfies (2.1) may be written in the form

$$
\phi=\sum_{n=0}^{\infty}\left[T_{n}\left(x_{2}\right) E_{n}\left(x_{1}+\tau\left(x_{2}\right)\right)+\bar{T}_{n}\left(x_{2}\right) \bar{E}_{n}\left(x_{1}+\bar{\tau}\left(x_{2}\right)\right],\right.
$$

where

$$
\begin{gathered}
T_{0}=(i)^{-1 / 2}\left(k_{11} k_{22}-k_{12}^{2}\right)^{-1 / 4} \alpha, \\
T_{n}=+\frac{1}{2}\left(k_{11} k_{22}-k_{12}^{2}\right)^{-1 / 4} \int\left[\frac{\left(d / d x_{2}\right)\left\{k_{22} T_{n-1}^{\prime}\right\}}{\left(k_{11} k_{22}-k_{12}^{2}\right)^{1 / 4}}\right] d x_{2} .
\end{gathered}
$$

Let $z=x_{1}+\tau\left(x_{2}\right)$ so that, from (3.2),

$$
E_{n}(z)=\int_{0}^{z} E_{n-1}(t) d t \text { for } n=1,2, \ldots
$$

Hence

$$
E_{n}(z)=\frac{1}{(n-1) !} \int_{0}^{z}(z-t)^{n-1} E_{0}(t) d t \text { for } n \geq 1
$$

If we choose

$$
E_{n}(z)=\frac{1}{2}(i)^{1 / 2} \Phi_{n}(z) \text { for } n=0,1,2,3, \ldots
$$

and $\alpha=1$, then (3.7) yields

$$
\phi=\mathscr{R}\left\{h_{0} \Phi_{0}(z)+\sum_{n=1}^{\infty} \frac{h_{n}\left(x_{2}\right)}{(n-1) !} \int_{0}^{z}(z-t)^{n-1} \Phi_{0}(t) d t\right\}
$$

where

$$
\begin{gathered}
h_{0}=\left(k_{11} k_{22}-k_{12}^{2}\right)^{-1 / 4} \\
h_{n}=\frac{1}{2}\left(k_{11} k_{22}-k_{12}^{2}\right)^{-1 / 4} \int\left[\frac{\left(d / d x_{2}\right)\left\{k_{22} h_{n-1}^{\prime}\right\}}{\left(k_{11} k_{22}-k_{12}^{2}\right)^{1 / 4}}\right] d x_{2} \quad \text { for } n \geq 1 .
\end{gathered}
$$

Eq. (3.13) provides the required solution to (2.1) in any domain in which the infinite series converges uniformly. The uniform convergence of the series may be investigated after the manner of Bergman [4], but here it will be suffieient to note that for certain inhomogeneities the series (3.13) truncates after a finite number of terms. In such cases (3.13) will certainly provide the required solution to (2.1). For example, the series terminates after one term if

$$
h_{0}^{\prime}=\frac{K}{k_{22}} \quad(K \text { constant })
$$

and after $n$ terms if $h_{n-1}^{\prime}=K / k_{22}$. In particular, if

$$
k_{i j}=\lambda_{i j} f\left(x_{2}\right) \text {, }
$$


where the $\lambda_{i j}$ are constants, then for the series to terminate after one term the function $f\left(x_{2}\right)$ must satisfy

$$
\left(\lambda_{11} \lambda_{22}-\lambda_{12}^{2}\right)^{-1 / 4} \frac{d}{d x_{2}}\left[f\left(x_{2}\right)\right]^{-1 / 2}=K / \lambda_{22} f\left(x_{2}\right)
$$

which yields

$$
f\left(x_{2}\right)=\left(C x_{2}+D\right)^{2}
$$

where $C$ and $D$ are arbitrary constants. Thus if

$$
k_{11}=\lambda_{11}\left(C x_{2}+D\right)^{2}, \quad k_{22}=\lambda_{22}\left(C x_{2}+D\right)^{2}, \quad k_{12}=\lambda_{12}\left(C x_{2}+D\right)^{2}
$$

then a general solution to (2.1) may be written in the form

$$
\phi=\Lambda\left(C x_{2}+D\right)^{-1} \mathscr{R}\left[\Phi_{0}(z)\right]
$$

where $\Lambda=\left(\lambda_{11} \lambda_{22}-\lambda_{12}\right)^{-1 / 4}$ and $\Phi_{0}(z)$ is an arbitrary analytic function. More generally, it may be verified that the series (3.13) terminates after a finite number of terms if the coefficients $k_{i j}$ are given by (3.17) with

$$
f\left(x_{2}\right)=\left(C x_{2}+D\right)^{2 N} \text { for } \quad N=0, \pm 1, \pm 2, \ldots
$$

\section{A reciprocal theorem}

THEOREM. Let $\phi$ be a solution of

$$
\frac{\partial}{\partial x_{i}}\left[k_{i j} \frac{\partial \phi}{\partial x_{j}}\right]=0
$$

valid in the region $R$ in $E^{2}$ bounded by the contour $C$ consisting of a finite number of piecewise smooth closed curves. Also let $\psi$ be another solution of (4.1) valid in $R$. Then

$$
\int_{c}\left[k_{i j} \frac{\partial \phi}{\partial x_{j}} n_{i} \psi-k_{i j} \frac{\partial \psi}{\partial x_{j}} n_{i} \phi\right] d s=0 .
$$

Proof:

$$
\begin{aligned}
\int_{C} k_{i j} \frac{\partial \phi}{\partial x_{j}} \psi n_{i} d s & =\int_{C}\left[k_{1 j} \frac{\partial \phi}{\partial x_{j}} \psi n_{i}+k_{2 j} \frac{\partial \phi}{\partial x_{j}} \psi n_{2}\right] d s \\
& =\int_{R}\left\{\frac{\partial}{\partial x_{1}}\left[k_{1 j} \frac{\partial \phi}{\partial x_{j}} \psi\right]+\frac{\partial}{\partial x_{2}}\left[k_{2 j} \frac{\partial \phi}{\partial x_{j}} \psi\right]\right\} d R
\end{aligned}
$$

and using (4.1)

$$
=\int_{R}\left\{k_{1 j} \frac{\partial \phi}{\partial x_{j}} \frac{\partial \psi}{\partial x_{1}}+k_{2 j} \frac{\partial \phi}{\partial x_{j}} \frac{\partial \psi}{\partial x_{2}}\right\} d R .
$$

Similarly,

$$
\int_{C} k_{i j} \frac{\partial \psi}{\partial x_{j}} \phi n_{i} d s=\int_{R}\left[k_{1 j} \frac{\partial \phi}{\partial x_{j}} \frac{\partial \psi}{\partial x_{1}}+k_{2 j} \frac{\partial \phi}{\partial x_{j}} \frac{\partial \psi}{\partial x_{2}}\right] d R .
$$

The required result follows immediately by subtraction of (4.4) from (4.3). 
5. The integral equation. In (4.2) let $\phi$ denote a required solution to a boundaryvalue problem governed by (2.1) and let $\psi$ be the solution to (2.1) given by (3.13) with

$$
\Phi_{0}(z)=\frac{1}{2 \pi} \log \left(z-z_{0}\right)
$$

where $z_{0}=a+\tau(b)$ with $(a, b)$ a point in $R$. Hence

$$
\begin{aligned}
\psi= & \frac{1}{2 \pi} \mathscr{R}\left\{h_{0} \log \left(z-z_{0}\right)+\sum_{n=1}^{\infty} \frac{h_{n}\left(x_{2}\right)}{(n-1) !} \int_{0}^{z}(z-t)^{n-1} \log \left(t-z_{0}\right) d t\right\} \\
= & \frac{1}{2 \pi} \mathscr{R}\left\{h_{0} \log \left(z-z_{0}\right)\right. \\
& +\sum_{n=1}^{\infty} \frac{h_{n}\left(x_{2}\right)}{(n-1) !} \sum_{r=0}^{n-1} \frac{(n-1) !(-1)^{r}}{r !(n-1-r) !} \frac{\left(z-z_{0}\right)^{n-1-r}}{r+1}\left\{\left(z-z_{0}\right)^{r+1} \log \left(z-z_{0}\right)\right. \\
& \left.-\left(-z_{0}\right)^{r+1} \log \left(-z_{0}\right)-(r+1)^{-1}\left(z-z_{0}\right)^{r+1}+(r+1)^{-1}\left(-z_{0}\right)^{r+1}\right\} .
\end{aligned}
$$

If (4.2) is to be valid with $\psi$ given by (5.2), then it is necessary to exclude the point $(a, b)$ by surrounding it with a small circle $\Gamma$ of radius $\varepsilon$. Then (4.2) yields

$$
\int_{C+\Gamma}\left[k_{i j} \frac{\partial \phi}{\partial x_{j}} n_{i} \psi-k_{i j} \frac{\partial \psi}{\partial x_{j}} n_{i} \phi\right] d s=0
$$

Now, on $\Gamma$,

$$
\begin{aligned}
x_{1}=a+\varepsilon & \cos \theta, \quad x_{2}=b+\varepsilon \sin \theta, \quad z_{0}=a+\tau(b), \\
z & =x_{1}+\tau\left(x_{2}\right) \\
& =a+\varepsilon \cos \theta+\tau(b+\varepsilon \sin \theta) \\
& =a+\varepsilon \cos \theta+\tau(b)+\varepsilon \sin \theta \tau^{\prime}(b)+O\left(\varepsilon^{2}\right), \\
z-z_{0} & =\varepsilon\left[\cos \theta+\tau^{\prime}(b) \sin \theta\right]+O\left(\varepsilon^{2}\right),
\end{aligned}
$$

so that

$$
\begin{aligned}
\psi= & \frac{1}{2 \pi} \mathscr{R}\left\{h_{0}(b+\varepsilon \sin \theta) \log \left[\varepsilon\left(\cos \theta+\tau^{\prime}(b) \sin \theta\right)+O\left(\varepsilon^{2}\right)\right]\right. \\
& +\sum_{n=1}^{\infty} \frac{h_{n}(b+\varepsilon \sin \theta)}{(n-1) !} \int_{0}^{z 0+\varepsilon \cos \theta+\varepsilon \tau^{\prime}(b) \sin \theta+O\left(\varepsilon^{2}\right)} \\
& \left.\cdot\left[z_{0}+\varepsilon \cos \theta+\varepsilon \sin \theta \tau^{\prime}(b)+O\left(\varepsilon^{2}\right)-t\right] \log \left(t-z_{0}\right) d t\right\} .
\end{aligned}
$$

Hence, on $\Gamma$, for small $\varepsilon$,

$$
\begin{gathered}
\psi=\frac{1}{2 \pi} \mathscr{R}\left\{h_{0}(b) \log \varepsilon\right\}+O(1), \\
\frac{\partial \psi}{\partial x_{1}}=\frac{1}{2 \pi} \mathscr{R}\left\{\frac{h_{0}(b)}{\varepsilon\left(\cos \theta+\sin \theta \tau^{\prime}(b)\right)}\right\}+O(\log \varepsilon), \\
\frac{\partial \psi}{\partial x_{2}}=\frac{1}{2 \pi} \mathscr{R}\left\{\frac{h_{0}(b) \tau^{\prime}(b)}{\varepsilon\left(\cos \theta+\sin \theta \tau^{\prime}(b)\right)}\right\}+O(\log \varepsilon) .
\end{gathered}
$$


Thus, for small $\varepsilon$,

$$
\begin{aligned}
\int_{\Gamma} k_{i j} & \frac{\partial \psi}{\partial x_{j}} \phi n_{i} d s \\
= & \frac{1}{2 \pi} \mathscr{R} \int_{0}^{2 \pi} \frac{h_{0}(b)\left[k_{11}(b) n_{1}+k_{12}(b) n_{1}+\left(k_{21}(b) n_{2}+k_{22}(b) n_{2}\right) \tau^{\prime}(b)\right]}{\cos \theta+\sin \theta \tau^{\prime}(b)} \\
& \cdot \phi(a, b) d \theta+O(\varepsilon \log \varepsilon) \\
= & -K(b) \phi(a, b)+O(\varepsilon \log \varepsilon),
\end{aligned}
$$

where (since $n_{1}=-\cos \theta, n_{2}=-\sin \theta$ )

$K(b)=\mathscr{R} \int_{0}^{2 \pi} \frac{h_{0}(b)\left\{\left[k_{11}(b)+k_{12}(b)\right] \cos \theta+\left[k_{21}(b)+k_{22}(b)\right] \tau^{\prime}(b) \sin \theta\right\}}{\cos \theta+\sin \theta \tau^{\prime}(b)} d \theta$.

Now from physical considerations the parameters $k_{i j}\left(x_{2}\right)$ are bounded in $R$, so that if the derivatives $\partial \phi / \partial x_{1}$ and $\partial \phi / \partial x_{2}$ are required to be bounded in $R$ then it follows that, for small $\varepsilon$,

$$
\int_{\Gamma} k_{i j} \frac{\partial \phi}{\partial x_{j}} n_{i} \psi d s=O(\varepsilon \log \varepsilon)
$$

Hence

$$
\lim _{\varepsilon \rightarrow \infty} \int_{\Gamma}\left[k_{i j} \frac{\partial \phi}{\partial x_{j}} n_{i} \psi-k_{i j} \frac{\partial \psi}{\partial x_{j}} n_{i} \phi\right] d s=K(b) \phi(a, b) .
$$

Thus (5.3) yields

$$
\phi(a, b)=\frac{-1}{K(b)} \int_{C}\left[k_{i j} \frac{\partial \phi}{\partial x_{j}} n_{i} \psi-k_{i j} \frac{\partial \psi}{\partial x_{i}} n_{i} \phi\right] d s .
$$

This equation is the required boundary integral equation for the solution of the boundary-value problem. If the point $(a, b)$ is on the boundary of the region $R$ then it is necessary to replace the formula (5.4) by

$K(b)=\mathscr{R} \int_{0}^{\alpha} \frac{h_{0}(b)\left\{\left[k_{11}(b)+k_{12}(b)\right] \cos \theta+\left[k_{21}(b)+k_{22}(b)\right] \tau^{\prime}(b) \sin \theta\right\}}{\cos \theta+\sin \theta \tau^{\prime}(b)} d \theta$.

The precise value of the angle $\alpha$ depends on the geometry of the boundary. If $C$ has a continuously turning tangent then $\alpha=\pi$.

In certain special cases it may be possible to employ (5.5) to obtain a simple analytical solution to a particular boundary-value problem. However, in general this will not be practical and Eq. (5.5) then forms the basis for an effective numerical method for the solution of the problem. The procedure for obtaining the numerical solution is as follows. If $k_{i j} \partial \phi / \partial x_{j} n_{i}$ is given on $C$ then the integral in (5.5) with $x_{0} \in C$ may be discretized to yield $m$ linear simultaneous equations for $\phi$ at $m$ points on the boundary $C$ of the region $R$. The integral in (5.5) with $(a, b) \in R$ may then be suitably discretized so that the righthand side of $(5.5)$ is known, and hence $\phi(a, b)$ may be determined for all $(a, b) \in R$. If, on the other hand, the $\phi(a, b)$ are given on $C$ then (5.5) may be discretized to yield $m$ simultaneous linear equations for the $k_{i j} \partial \phi / \partial x_{j} n_{i}$ at $m$ points on $C$. Eq. (5.5) with $(a, b) \in R$ then yields $\phi(a, b)$. Mixed boundary data may be handled in a similar manner. 


\section{REFERENCES}

[1] T. A. Cruse and J. C. Lachat (eds.), Proceedings of the International Symposium on Innovative Numerical Analysis in Applied Engineering Science (Versailles, France, 1977)

[2] T. A. Cruse and F. J. Rizzo (eds.), Boundary integral equation method: Computational applications in applied mechanics (ASME Proceedings, AMD, Vol. II, 1975)

[3] D. L. Clements, $A$ boundary integral equation method for the numerical solution of a second order elliptic equation with variable coefficients, J. Aust. Math. Soc. (Series B) 22, 218-228 (1980)

[4] S. Bergman, Integral operators in the theory of linear partial differential equations, Springer-Verlag, 1971

[5] D. L. Clements, Boundary value problems governed by second order elliptic systems, Pitman, 1981 